Research article

Open Access

\title{
Differential expression of RANK, RANK-L, and osteoprotegerin by synovial fluid neutrophils from patients with rheumatoid arthritis and by healthy human blood neutrophils
}

Patrice E Poubelle, Arpita Chakravarti, Maria J Fernandes, Karine Doiron and Andrée-

Anne Marceau

\begin{abstract}
Centre de Recherche en Rhumatologie et Immunologie, Centre de Recherche du Centre Hospitalier de l'Université Laval (CRCHUL), 2705 boulevard Laurier, Ste-Foy, QC G1V 4G2, Canada
\end{abstract}

Corresponding author: Patrice E Poubelle, Patrice.Poubelle@crchul.ulaval.ca

Received: 26 Oct 2006 Revisions requested: 4 Jan 2007 Revisions received: 9 Feb 2007 Accepted: 6 Mar 2007 Published: 6 Mar 2007

Arthritis Research \& Therapy 2007, 9:R25 (doi:10.1186/ar2137)

This article is online at: http://arthritis-research.com/content/9/2/R25

(c) 2007 Poubelle et al.; licensee BioMed Central Ltd.

This is an open access article distributed under the terms of the Creative Commons Attribution License (http://creativecommons.org/licenses/by/2.0), which permits unrestricted use, distribution, and reproduction in any medium, provided the original work is properly cited.

\begin{abstract}
Functional links between bone remodeling and the immune system in chronic inflammatory arthritis are mediated, in part, by the ligand of receptor activator of nuclear factor-kappa-B (RANK-L). Because neutrophils play a crucial role in chronic inflammation, the goal of this study was to determine whether proteins of the RANK/RANK-L pathway are expressed by synovial fluid (SF) neutrophils from patients with rheumatoid arthritis (RA) and to characterize this pathway in normal human blood neutrophils. The expression of RANK-L, osteoprotegerin (OPG), RANK, and tumor necrosis factor receptor-associated factor 6 (TRAF6) was determined by polymerase chain reaction, enzyme-linked immunosorbent assay, Western blotting, and cytofluorometry. RANK signaling was analyzed by the degradation of inhibitor of kappaB-alpha (I-kB- $\alpha)$. SF neutrophils from patients with RA express and release OPG and express the membrane-associated forms of RANK-L and RANK. In contrast, normal blood neutrophils express only the membrane-associated form of RANK-L. They do not express the
\end{abstract}

mRNAs encoding OPG and RANK. SF neutrophils from RA patients and normal blood neutrophils release no soluble RANKL. They express the mRNA for TRAF6. The expression of OPG and RANK by normal human blood neutrophils, however, can be induced by interleukin- 4 + tumor necrosis factor-alpha and by SFs from patients with RA. In contrast, SFs from patients with osteoarthritis do not induce the expression of OPG and RANK. Moreover, the addition of RANK-L to normal blood neutrophils pretreated by SF from patients with RA decreased $\mathrm{I}-\kappa \mathrm{B}-\alpha$, indicating that RANK signaling by neutrophils stimulated with SF is associated with nuclear factor-kappa-B activation. In summary, RANK-L is expressed by inflammatory and normal neutrophils, unlike OPG and RANK, which are expressed only by neutrophils exposed to an inflammatory environment. Taken together, these results suggest that neutrophils may contribute to bone remodeling at inflammatory sites where they are present in significantly large numbers.

\section{Introduction}

Neutrophils, which are among the first cells to arrive in inflamed tissues, are activated during their margination and diapedesis across blood vessels and by cytokines at the site of inflammation [1]. They are involved in various chronic inflammatory diseases such as arthritis, active autoimmune colitis, and skin lesions of psoriasis [2,3]. In rheumatoid arthritis (RA), neutrophils are found in synovial fluids (SFs) and at the rheumatoid pannus-cartilage junction. They can degrade cartilage constituents $[4,5]$. The essential role of neutrophils in the initiation and maintenance of inflammation in the affected

\footnotetext{
$\mathrm{Ab}=$ antibody; $\mathrm{BSA}=$ bovine serum albumin; $\mathrm{CM}=$ control medium; EIA = enzyme immunometric assay; ELISA = enzyme-linked immunosorbent assay; FBS = fetal bovine serum; FITC = fluorescein isothiocyanate; GM-CSF = granulocyte-macrophage colony-stimulating factor; HBSS $=$ Hanks' balanced salt solution; HRP = horseradish peroxidase; $\mathrm{Ig}=$ immunoglobulin; I- $\mathrm{\kappa B}-\alpha=$ inhibitor of kappaB-alpha; $I \mathrm{~L}=$ interleukin; $\mathrm{LDH}=$ lactate dehydrogenase; $\mathrm{MHC}=$ major histocompatibility complex; $\mathrm{NF}-\mathrm{\kappa B}=$ nuclear factor-kappa-B; $\mathrm{OA}=$ osteoarthritis; $\mathrm{OPG}=$ osteoprotegerin; $\mathrm{PBML}=$ peripheral blood mononuclear leukocyte; $P C R=$ polymerase chain reaction; $P V D F=$ polyvinylidene difluoride; $R A=$ rheumatoid arthritis; $R A N K=$ receptor activator of nuclear factor-kappa-B; RANK-L = ligand of receptor activator of nuclear factor-kappa-B; $R F=$ rheumatoid factor; $R T-P C R=$ reverse transcriptase-polymerase chain reaction; $S D=$ standard deviation; $S E M=$ standard error of the mean; $S F=$ synovial fluid; $S M=$ survival medium; TBS $=$ tris-buffered saline; TNF $=$ tumor necrosis factor; TRAF6 $=$ tumor necrosis factor receptor-associated factor 6 ; TRANCE $=$ tumor necrosis factorrelated activation-induced cytokine.
} 
joints in RA was confirmed by the $\mathrm{K} / \mathrm{BxN}$ mouse model of RA [6].

Besides their role in innate immunity, neutrophils act as antigen-presenting cells and regulate the adaptive immune response [7]. In the presence of certain cytokines, neutrophils acquire a variety of biological characteristics - such as the expression of major histocompatibility complex (MHC) class II antigens - that enable them to function as antigen-presenting cells $[8,9]$. In addition, phlogogenic cytokines activate neutrophils to express CCR6, CD80, CD83, CD86, and CD40, an expression pattern that resembles a dendritic-like phenotype $[10,11]$. The in vitro observation that neutrophils differentiate into dendritic-like cells has been corroborated in vivo by the demonstration that they express MHC class II, CD80, and CD86 proteins and that they can present antigens to T cells in an $\mathrm{MHC}$ class II-restricted manner in Wegener granulomatosis and RA $[12,13]$. The same inflammatory conditions that induce neutrophils to differentiate into dendritic-like cells have the capacity to delay the apoptosis of neutrophils, which are cells that are constitutively programmed for apoptotic cell death [14].

During the immune response, mature dendritic cells express receptor activator of nuclear factor-kappa-B (RANK) and tumor necrosis factor receptor-associated factor 6 (TRAF6) $[15,16]$. TRAF6 is an adapter protein implicated in signaling pathways of immunity and bone homeostasis [16]. RANK is activated by tumor necrosis factor-related activation-induced cytokine (TRANCE) [15]. TRANCE is a new member of the tumor necrosis factor (TNF) family and prevents apoptosis, increases survival, and stimulates cytokine production in dendritic cells $[17,18]$. TRANCE and the ligand of RANK (RANK$\mathrm{L})$ were originally cloned and sequenced from $T$ lymphocytes $[15,17]$. They are also known as osteoprotegerin (OPG) ligand and osteoclast differentiation factor based on their capacity to induce osteoclastogenesis and activate osteoclasts via RANK [19-21]. Bone resorption is dependent upon osteoblast-osteoclast interactions that are mediated through the osteoblastic expression of a membrane form of RANK-L. This protein can also be processed into a soluble and active extracellular form [22]. In the presence of certain stimuli, cells can express both RANK-L and RANK, an observation reported in T lymphocytes $[15,23]$. These studies have shed light on the molecular and functional links between bone remodeling and the immune system. T lymphocytes, for instance, promote bone loss in inflammatory arthritis by expressing RANK-L that directly binds and activates osteoclasts [24].

The observation that neutrophils can differentiate into dendritic-like cells led us to test the hypothesis that inflammatory neutrophils could express proteins common to the local immune response and bone remodeling, such as those of the RANK/RANK-L pathway. To address this question, we investigated the expression of RANK-L, OPG, RANK, and TRAF6
mRNAs and proteins in neutrophils from the SF of patients with RA. Human blood neutrophils from healthy subjects were studied as normal control cells. Moreover, we demonstrate that the expression of genes of the RANK/RANK-L pathway could be induced by certain stimuli in neutrophils in vitro. The effect of SFs from patients with RA and from patients with osteoarthritis (OA) on the expression of these genes by normal neutrophils was also evaluated. Our observations suggest that the proteins of the RANK/RANK-L pathway expressed by neutrophils mediate important functions of neutrophils during the abnormal immune response and bone remodeling in RA.

\section{Materials and methods Reagents}

Ficoll-Paque (1.077 density), RPMI 1640, Hanks' balanced salt solution (HBSS), and fetal bovine serum (FBS) were purchased from WISENT Inc. (St-Bruno, QC, Canada). Terminal deoxynucleotidyl transferase was purchased from Amersham Biosciences Inc. (now part of GE Healthcare, Little Chalfont, Buckinghamshire, UK). Trizol reagents and the Superscript ${ }^{\mathrm{TM}} \|$ Reverse Transcriptase (RT) kit were obtained from Invitrogen Corporation (Carlsbad, CA, USA). Oligo-dT primers and Taq DNA polymerase were purchased from PerkinElmer Life and Analytical Sciences (Woodbridge, ON, Canada). The goat polyclonal anti-human RANK-L immunoglobulin (lg) $\mathrm{G}$ antibody (Ab) (sc-7627) was purchased from Santa Cruz Biotechnology, Inc. (Santa Cruz, CA, USA). The mouse monoclonal anti-human RANK $\mathrm{Ab}$ was obtained from Alexis Biochemicals (part of Axxora Life Sciences, Inc., San Diego, CA, USA). The rabbit polyclonal anti-human inhibitor of kappaB-alpha $\mathrm{Ab}$ (no. 9242) was purchased from Cell Signaling Technology, Inc. (Danvers, MA, USA). Human recombinant RANK-L was obtained from PeproTech (Rocky Hill, NJ, USA). The human RANK cDNA was a kind gift from Dr. Naoki Sakurai (Discovery Research Laboratory, Tanabe Seiyaku Co., Ltd., Yodogawaku, Osaka, Japan).

\section{Cell preparation and culture conditions}

The institutional review board of the Université Laval (Québec, QC, Canada) approved the present study, and volunteers signed a consent form. Samples were collected in anticoagulant solution, and cells were isolated under sterile conditions. Cells were obtained from the human venous blood of healthy donors and from the SF of seven patients with RA (according to the revised criteria of the American College of Rheumatology). Characteristics of patients with RA were as follows: six women/one man, age at onset of symptoms $52.1 \pm 16.2$ years (mean \pm standard deviation [SD]), time between onset of symptoms and the present study $4.6 \pm 4.0$ years (mean \pm SD), clinical parameters at the present examination: erythrocyte sedimentation rate (ESR) $27.6 \pm 15.6 \mathrm{~mm}$ (mean $\pm \mathrm{SD}$ ), and C-reactive protein $36.2 \pm 31.7 \mathrm{~g} / \mathrm{l}$ (mean $\pm \mathrm{SD}$ ). Four patients were positive for IgM-rheumatoid factor (RF), and three were negative for IgM-RF. Four patients had radiographic erosions, two had local osteoporosis of inflammatory joints, and one 
showed no radiographic symptoms. Three patients had undergone no treatment, one was taking non-steroidal anti-inflammatory drugs, one was taking $5 \mathrm{mg} /$ day prednisone, and two were taking disease-modifying anti-rheumatic drugs. Due to limited quantities of SF and neutrophils from patients with RA and due to the requirement of large numbers of cells depending on the experiments performed (described below), it was not possible to systematically include the cells of the seven patients in all the experiments reported.

Blood was centrifuged ( $250 \mathrm{~g}, 15$ minutes) and the plateletrich plasma was removed. The peripheral blood polymorpho (neutrophils) and mononuclear leukocyte (PBML) fractions were obtained by centrifugation over Ficoll-Paque after dextran sedimentation [25]. Remaining erythrocytes were eliminated by hypotonic lysis. SF neutrophils were directly obtained by centrifugation over Ficoll-Paque. After two washes, cells were counted and resuspended in culture medium. Differential cell counts of leukocytes were performed by cytofluorometry (EPICS-XL; Beckman Coulter, Fullerton, CA, USA) and Wright's and non-specific esterase stains. Neutrophil suspensions were more than 98\% pure with no CD3-positive cells, and non-specific esterase-positive cells represented less than $0.2 \%$ of the cell population.

Cells were incubated in 12 -well plates $(2 \mathrm{ml} /$ well $)$ at $37^{\circ} \mathrm{C}$ and $5 \% \mathrm{CO}_{2}$ for up to 4 days. Two culture media were studied. The control medium (CM) was RPMI 1640 and 10\% FBS, and the survival medium (SM) consisted of $\mathrm{CM}$ supplemented with $500 \mathrm{pM}$ granulocyte-macrophage colony-stimulating factor (GM-CSF), $10 \mathrm{ng} / \mathrm{ml}$ interleukin (IL)-4, and $10 \mathrm{ng} / \mathrm{ml} \mathrm{TNF-} \alpha$. The cytokines present in SM were chosen for their anti-apoptotic effects on neutrophils $[10,26,27]$. Cells and supernatants were collected from days 1 to 4 . After centrifugation $(5,000 \mathrm{~g}$, 2 minutes), cell pellets were resuspended in $1 \mathrm{ml}$ of Trizol for RNA isolation or sonicated in $0.5 \mathrm{ml}$ of HBSS (no. 211-512) for enzyme immunometric assay (EIA) analysis of cell-associated materials. These samples were frozen at $-20^{\circ} \mathrm{C}$ until assayed. When required, samples of neutrophil supernatants were concentrated by centrifugation over Amicon Ultra 10000 MW CO (Millipore Corporation, Billerica, MA, USA) at 5,000 $g$ for 1 hour at $4^{\circ} \mathrm{C}$. Normal peripheral blood neutrophils $\left(10^{7 /}\right.$ $\mathrm{ml}$ ) were also incubated at $37^{\circ} \mathrm{C}$ and $5 \% \mathrm{CO}_{2}$ for 3 days in the presence of acellular SF from four of the seven RA patients described above and in the presence of acellular SF from two patients with OA. The incubation media consisted of $80 \% \mathrm{SF}$ and $20 \% \mathrm{CM}$.

\section{Analysis by reverse transcriptase-polymerase chain reaction}

Total RNA was isolated from cells by means of the Trizol reagent, and RT reaction was performed with Superscript ${ }^{\mathrm{TM}}$ II RT according to the manufacturer's instructions. The CDNAs were amplified by polymerase chain reaction (PCR) using genespecific primer pairs designed with Primer 3 software (White- head Institute for Biomedical Research, Cambridge, MA, USA) (Table 1). Each PCR was performed with one tenth of the volume of cDNA from the RT reaction, $10 \mu \mathrm{M}$ forward and reverse primers, $200 \mu \mathrm{M}$ dNTPs, $2.5 \mu \mathrm{l} 10 \times \mathrm{PCR}$ buffer (200 $\mathrm{mM}$ Tris- $\mathrm{HCl} \mathrm{pH} 8.4,500 \mathrm{mM} \mathrm{KCl}), 1$ to $1.5 \mathrm{mM} \mathrm{MgCl}_{2}, 0.5 \mathrm{U}$ Taq DNA polymerase, and autoclaved, distilled water to obtain a final volume of $25 \mu \mathrm{l}$. The number of cycles corresponding to the linear phase of amplification and the annealing temperature were optimized for each primer set (Table 1). The human $\beta$-actin transcript was used to standardize between PCRs. The PCR products were separated on a $1 \%$ agarose gel by electrophoresis in Tris acetic acid EDTA (ethylenediaminetetraacetic acid) buffer and visualized using ethidium bromide. The sequence of the amplified gene fragments was determined by direct sequencing.

\section{EIA analysis of RANK-L and OPG}

The EIAs used were at two sites with horseradish peroxidase (HRP) as a tracer. Ninety-six-well plates were coated with either the human OPG/Fc Chimera (805-OS; R\&D Systems, Inc., Minneapolis, MN, USA) or a monoclonal anti-human OPG Ab (MAB8051; R\&D Systems, Inc.) in phosphate-buffered solution ( $\mathrm{pH}$ 7.4). A biotinylated secondary goat anti-human RANK-L Ab (BAF626; R\&D Systems, Inc.) or a compatible biotinylated secondary goat anti-human OPG Ab (BAF805; R\&D Systems, Inc.) in phosphate-buffered solution ( $\mathrm{pH} 7.4)$ containing bovine serum albumin (BSA) was used. Antigen-Ab complexes were detected by the addition of a streptavidinHRP conjugate and tetramethylbenzidine as a substrate for HRP. Concentrations of RANK-L and OPG were obtained from a standard curve generated by known concentrations of human RANK-L and OPG. The detection limits were 15 and $7.5 \mathrm{pg} / \mathrm{ml}$ for RANK-L and OPG, respectively.

\section{Western blot analysis}

SF neutrophils $\left(3 \times 10^{6}\right)$ from four patients with RA were solubilized in SDS sample buffer. The positive control was COS7 cells transiently transfected (Fugene 6 transfection reagent; Roche Diagnostics, Indianapolis, IN, USA) with human RANK cDNA. Transfected cells were lysed in $1.5 \%$ Triton X-100 at $4^{\circ} \mathrm{C}$ for 5 minutes, and samples were analyzed on a $7 \%$ SDSpolyacrylamide gel. The proteins were transferred to a polyvinylidene difluoride (PVDF) membrane (Millipore Corporation) at $4^{\circ} \mathrm{C}$ overnight. The membrane was blocked with $2 \%$ pig gelatin in tris-buffered saline (TBS) with $6 \%$ Tween 20 for 60 minutes, incubated with $0.2 \mu \mathrm{g} / \mathrm{ml}$ of a goat anti-human RANK IgG $A b$ (no. AF683; R\&D Systems, Inc.), washed three times in TBS-Tween, and incubated with $0.04 \mu \mathrm{g} / \mathrm{ml}$ of a rabbit HRPconjugated anti-goat IgG Ab (The Jackson Laboratory, Bar Harbor, ME, USA). After incubation in SF from patients with RA for 3 days (see above), healthy blood neutrophils were centrifuged at $600 \mathrm{~g}$ for 30 minutes on a percoll gradient to remove debris and dead cells [28], washed, resuspended in HBSS ( $15 \times 10^{6}$ cells per milliliter), and stimulated at $37^{\circ} \mathrm{C}$ by $50 \mathrm{ng} / \mathrm{ml}$ TNF- $\alpha$ for 10 minutes or by $100 \mathrm{ng} / \mathrm{ml}$ RANK-L for 
Table 1

DNA sequences of the forward and reverse primers for the qualitative and semi-quantitative reverse transcriptase-polymerase chain reaction analyses

\begin{tabular}{|c|c|c|c|c|c|c|}
\hline Gene identity & $\begin{array}{l}\text { Accession } \\
\text { number }\end{array}$ & Primer sequences ${ }^{a}$ & $\begin{array}{l}\text { Annealing } \\
\text { temperature }\left({ }^{\circ} \mathrm{C}\right)\end{array}$ & $\begin{array}{l}\text { Number of cycles } \\
\text { (Quan.) }\end{array}$ & $\begin{array}{l}\text { Number of cycles } \\
\text { (Qual.)c }\end{array}$ & $\begin{array}{l}\text { Size of PCR product } \\
\text { (bp) }\end{array}$ \\
\hline \multirow[t]{2}{*}{$R A N K-L$} & AF019047 & 5'-CTG-ATG-AAA-GGA-GGA-AGC-AC-3' & 65 & 29 & 35 & 546 \\
\hline & & 5'-GAT-GAC-ACC-CTC-TCC-ACT-TC-3' & & & & \\
\hline \multirow[t]{2}{*}{$O P G$} & U94332 & 5'-TGC-TGT-TCC-TAC-AAA-GTT-TAC-G-3' & 56 & 35 & 40 & 433 \\
\hline & & 5'-CTT-TGA-GTG-CTT-TAG-TGC-GTG-3' & & & & \\
\hline \multirow[t]{2}{*}{ RANK } & AF018253 & 5'-CCT-GGA-CCA-ACT-GTA-CCT-TC-3' & 58 & 34 & 40 & 500 \\
\hline & & 5'-TTC-CTC-TAT-CTC-GGT-CTT-GC-3' & & & & \\
\hline \multirow[t]{2}{*}{ TRAF6 } & U78798 & 5'-TGA-TAG-TGT-GGG-TGG-AAC-TG-3' & 58 & 27 & 35 & 456 \\
\hline & & 5'-CTC-CTT-GGA-CAA-TCC-TTC-AG-3' & & & & \\
\hline \multirow[t]{2}{*}{$\beta$-Actin } & NM001101 & $\begin{array}{l}\text { 5'-CGT-GAC-ATT-AAG-GAG-AAG-CTG- } \\
\text { TGC-3' }\end{array}$ & 58 & 21 & 28 & 375 \\
\hline & & $\begin{array}{l}\text { 5'-CTC-AGG-AGG-AGC-AAT-GAT-CTT- } \\
\text { GAT-3' }\end{array}$ & & & & \\
\hline
\end{tabular}

aFor each pair of sequences, the forward primer appears first and the reverse primer appears second. bThe number of cycles used in the semiquantitative (quan.) PCR experiments corresponds to the linear phase of the amplification reaction. ${ }^{c}$ The number of cycles used in the qualitative (qual.) PCR experiments was increased to allow the possible detection of the mRNA. OPG, osteoprotegerin; PCR, polymerase chain reaction; RANK, receptor activator of nuclear factor-kappa-B; RANK-L, ligand of receptor activator of nuclear factor-kappa-B; TRAF6, tumor necrosis factor receptor-associated factor 6 .

10 and 20 minutes. They were then transferred to $2 \times$ boiling Laemmli's sample buffer ( $1 \times$ : $62.5 \mathrm{mM}$ Tris/HCl [pH6.8], 4\% [wt/vol] SDS, 5\% [vol/vol] 2-mercaptoethanol, 8.5\% [vol/vol] glycerol, $2.5 \mathrm{mM}$ orthovanadate, $10 \mathrm{mM}$ para-nitrophenylphosphate, $10 \mu \mathrm{g} / \mathrm{ml}$ leupeptin, $10 \mu \mathrm{g} / \mathrm{ml}$ aprotinin, and $0.025 \%$ bromophenol blue). Proteins were separated on a $12 \%$ SDSPAGE gel and transferred on a PVDF membrane. Immunoblotting was performed using $5 \%$ Blotto as a blocking agent. The primary $\mathrm{Ab}$ directed against $\mathrm{I}-\mathrm{KB}-\alpha$ was diluted $1: 1,000$ in TBS-Tween 5\% BSA and incubated with the membrane for 1 hour. The goat HRP-conjugated anti-rabbit IgG Ab (The Jackson Laboratory) was diluted 1:20,000 and incubated with the membrane. The labeled Abs were detected by the ECL (enhanced chemiluminescence) detection system (GE Healthcare) and visualized on Kodak Biomax MR film (Eastman Kodak, Rochester, NY, USA).

\section{Cytofluorometry}

The expression of RANK-L and RANK at the membrane was evaluated by cytofluorometry. Freshly separated healthy human blood or SF neutrophils from patients with RA and healthy neutrophils incubated in SFs were incubated with a goat anti-human RANK-L Ab (Santa Cruz Biotechnology, Inc.) followed by a fluorescein isothiocyanate (FITC)-conjugated anti-goat $F\left(a b^{\prime}\right)_{2} A b$. A normal goat lgG was used as control. To evaluate cell surface expression of RANK, healthy human blood neutrophils incubated in SFs were fixed and permeabilized using the Fixation/Permeabilization Solution kit (no. 554723) from BD Biosciences Pharmingen (San Diego, CA, USA). Briefly, non-specific staining of Fc receptors was blocked by $10 \%$ human decomplemented serum before cells were resuspended in $250 \mu$ l of Fixation/Permeabilization Solu- tion (BD Cytofix/Cytoperm no. 554714) for 45 minutes at $4^{\circ} \mathrm{C}$. Cells were then washed and permeabilized with BD Perm/ Wash buffer in the presence of $10 \%$ mouse non-specific immune serum. Fixed and permeabilized neutrophils were then stained with a mouse monoclonal anti-human RANK Ab (ALX804-212-C100) followed by a FITC-conjugated anti-mouse $\left.\mathrm{F}(\mathrm{ab})_{2}\right)_{2} \mathrm{Ab}$. Corresponding controls with non-specific Abs were also performed.

\section{Viability}

Neutrophil viability was evaluated by the lactate dehydrogenase (LDH) release assay. Neutrophil suspensions after incubation were centrifuged (5,000 g, 1 minute). Supernatants and pelleted neutrophils were collected separately, and cells were lysed in 1\% Triton X-100 buffer. Prior to colorimetric analysis at $340 \mathrm{~nm}, 1.25 \mathrm{ml}$ of substrate $(0.14 \mathrm{mg} / \mathrm{ml} \mathrm{NADH}$ in $0.1 \mathrm{M}$ sodium phosphate buffer, $\mathrm{pH} 7.35$ ) and $50 \mu \mathrm{l}$ of pyruvate solution were added to $50 \mu$ l of cell lysate or supernatant. Results were expressed as percentages of the ratio between the optical density values measured in supernatants and the total optical density value measured in cells plus supernatants. Viable neutrophils that did not release LDH at day 3 represented $32 \%$ and $39 \%$ in CM and SM, respectively.

\section{Statistics}

Values were expressed as means \pm standard error of the mean (SEM) of $n$ experiments performed with cells from different donors. Statistical analyses were performed using GraphPad Instat 3.0 (GraphPad Software, Inc., San Diego, CA, USA). Non-parametric analysis with the Mann-Whitney test was used to compare the means of two groups. Paired groups were ana- 
lyzed using the paired $t$ test. Significance was set at a twotailed $p$ value of less than 0.05 .

\section{Results}

Expression of RANK-L, OPG, RANK, and TRAF6 mRNAs by SF neutrophils from patients with RA and by healthy human blood cells

Freshly isolated neutrophils from SF of patients with RA expressed RANKL, OPG, TRAF6, and RANK as determined by semi-quantitative RT-PCR (Figure 1a). In contrast, freshly isolated peripheral blood neutrophils from healthy subjects expressed RANK-L and TRAF6, but not OPG and RANK (Figure 1a). PBMLs from healthy subjects expressed the four genes tested, and platelets expressed RANK-L, TRAF6, OPG, and not RANK (Figure 1b). The absence of OPG or RANK expression in healthy neutrophils was confirmed by qualitative PCR using an increasing number of cycles as indicated in Table 1 (data not shown). The fact that PBMLs expressed
OPG and RANK mRNA enabled us to confirm that the neutrophil and platelet preparations were not contaminated by these cells.

\section{Expression of RANK-L, OPG, and RANK proteins by SF neutrophils from patients with RA}

Freshly isolated neutrophils from SF of patients with RA expressed not only the mRNA of the four genes studied (Figure 1a) but also the corresponding proteins RANK-L, OPG, and RANK (Figure 2). Cell-associated materials of SF neutrophils from patients with RA contained detectable amounts of RANK-L and OPG as measured by EIAs (Figure 2a,c). In contrast, cell-associated materials of healthy blood neutrophils contained $68 \pm 13 \mathrm{pg} / \mathrm{ml}$ RANK-L and no OPG $(n=13)$. SF neutrophils obtained from patients with RA and incubated for up to 4 days in CM (as described in Materials and methods and Figure 2a) or in SM (data not shown) did not release

Figure 1
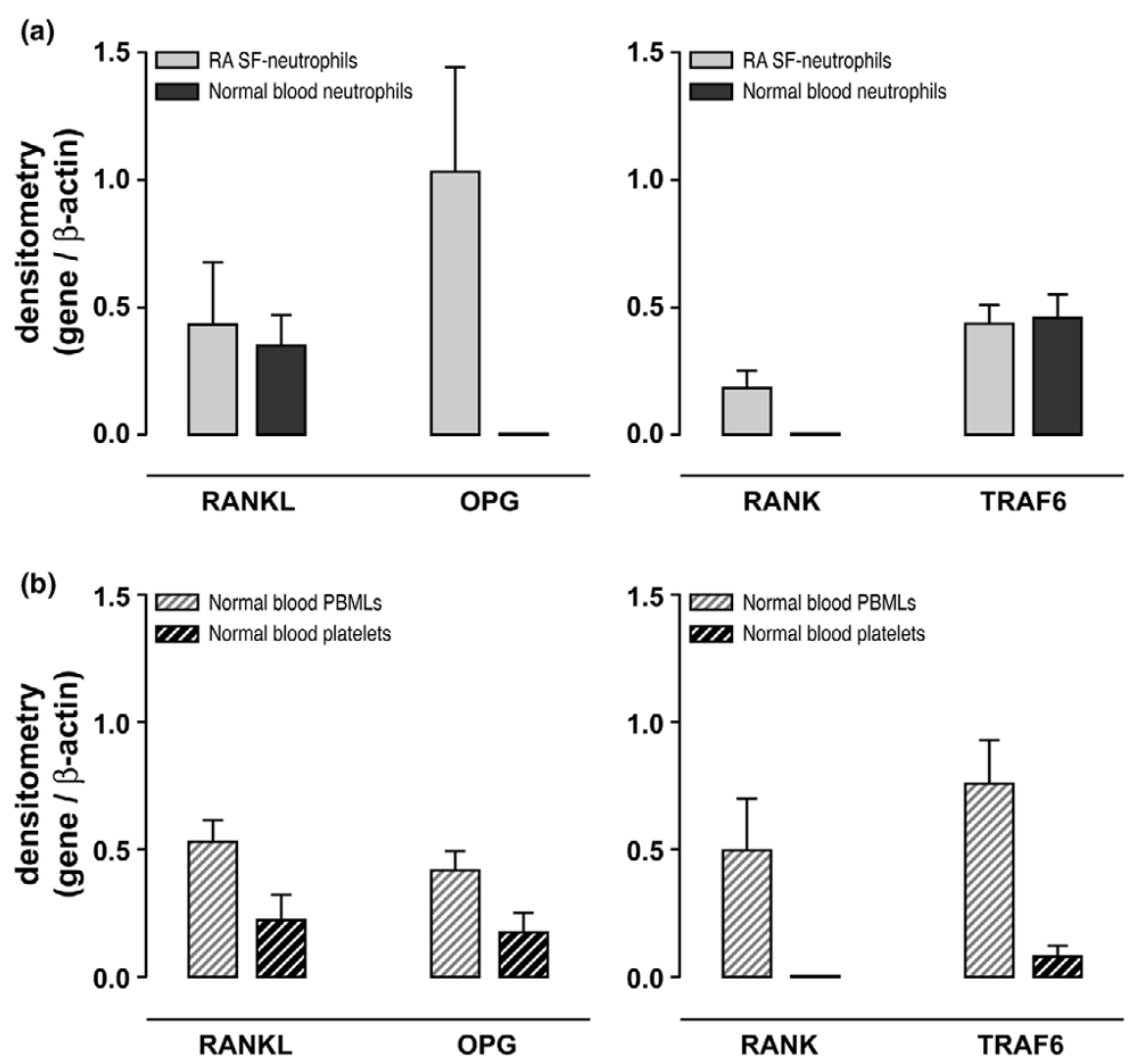

Expression of RANK-L, OPG, RANK, and TRAF6 mRNAs by synovial fluid (SF) neutrophils isolated from patients with rheumatoid arthritis (RA) and by normal blood cells. (a) Purified neutrophils of SF from four patients with RA and of blood from seven normal donors were evaluated by semi-quantitative reverse transcriptase-polymerase chain reaction (RT-PCR) analyses. (b) Purified peripheral blood mononuclear leukocytes (PBMLs) and platelets of blood from seven normal donors were evaluated by semi-quantitative RT-PCR analyses. To avoid leukocyte contamination in the platelet suspension, platelets were isolated from the upper part of the platelet-rich plasma (PRP). After centrifugation (600 $\mathrm{g}, 30 \mathrm{minutes})$, the pellets were resuspended in Trizol. No contaminating leukocytes in the upper part of the PRP were observed under light microscopy after Wright's stain. Histograms represent mean \pm standard error of the mean of ratios of densitometric values for RANK-L/ $\beta$-actin, OPG/ $\beta$-actin, RANK/ $\beta$-actin, and TRAF6/ $\beta$-actin. OPG, osteoprotegerin; RANK, receptor activator of nuclear factor-kappa-B; RANK-L, ligand of receptor activator of nuclear factor-kappa-B; TRAF6, tumor necrosis factor receptor-associated factor 6 . 
RANK-L. However, freshly isolated SF neutrophils of patients with RA, as well as healthy blood neutrophils, expressed RANK-L protein on their plasma membrane, as evaluated by cytofluorometry (Figure 2b). Percentages of RANK-L-positive neutrophils freshly isolated from SF of patients with RA $(n=5)$ and from normal blood $(n=13)$ were $10.9 \% \pm 4.3 \%$ and $2.9 \%$ $\pm 0.7 \%$, respectively $(p=0.09)$. Moreover, the same SF neutrophils obtained from patients with RA and incubated for up to 4 days in $C M$ showed a time-dependent release and accumulation of OPG in supernatants (Figure 2c). Finally, the expression of RANK protein was also determined by Western blot analysis in freshly isolated SF neutrophils of four patients with RA. The SF neutrophils of two of the four patients studied expressed a detectable band of an apparent molecular weight of $110 \mathrm{kDa}$. This band is specific for the polyclonal anti-human RANK $A b$ as shown in COS cells transiently transfected with a human RANK cDNA (Figure 2d). The amounts of OPG and RANK-L in SFs of the same patients with RA were also quantitated by EIA. SFs of patients with RA contained 6,990 \pm $1,912 \mathrm{pg} / \mathrm{ml}$ OPG and $21 \pm 12 \mathrm{pg} / \mathrm{ml}$ RANK-L (mean \pm SEM, $n=7$ ) with a RANK-L/OPG ratio of 0.003 .

\section{Normal human blood neutrophils can acquire the capacity to express OPG and RANK}

We next investigated whether in vitro conditions could mimic our in vivo observations (Figures 1 and 2). Neutrophils were incubated with cytokines that decrease neutrophil apoptosis and that are found in SFs from patients with RA [29]. Healthy blood neutrophils were shown to express RANK-L mRNA under $\mathrm{CM}$ and $\mathrm{SM}$ conditions without any significant changes from day 1 to day 3 (Figure 3). The expression of OPG mRNA by neutrophils incubated in $\mathrm{CM}$ was not yet detectable on day 2 and appeared only after 3 days (Figure 3 ). The incubation of neutrophils in SM, however, strikingly upregulated the expression of this gene. The expression of OPG mRNA, which was absent at day 0 (Figure 1a), significantly increased at days 1 , 2 , and 3 (Figure 3 ). Control studies using different combinations of the cytokines were also conducted. Neutrophils incubated for 3 days in medium containing TNF- $\alpha$ alone expressed RANK-L but not OPG. When IL-4 was present, alone or in combination with GM-CSF or TNF- $\alpha$, neutrophils expressed OPG with no change of RANK-L. GM-CSF alone had no effect on the expression of the genes tested (data not shown). Healthy blood neutrophils that do not express RANK at day 0 (Figure 1a) have the capacity to express RANK mRNA in vitro when incubated in SM (Figure 3). The expression of RANK by neutrophils was detectable from day 2 to day 3 (results observed in two donors out of nine healthy subjects studied). The expression of TRAF6 by normal blood neutrophils, on the other hand, was detected in all the conditions tested but decreased significantly at day 3 in the presence of SM (Figure 3). In contrast, PBMLs expressed OPG and RANK from day 0 (Figure $1 \mathrm{~b}$ ) to day 3 (data not shown). In these cells, a decrease in the expression of OPG and RANK was observed when incubated in $\mathrm{CM}$ and an increase was observed when incubated in SM. TRAF6 expression by PBMLs was similar in $\mathrm{CM}$ and in SM with no significant changes from days 1 to 3 (data not shown).

These findings were then confirmed at the protein level. Incubation of healthy blood neutrophils in $\mathrm{CM}$ or SM conditions for up to 3 days did not modify the membrane expression of RANK-L as evaluated by cytofluorometry and did not stimulate the release of detectable amounts of OPG in neutrophil supernatants as measured by EIA (data not shown). Prolongation of the incubation time up to 5 days in SM, however, led to an accumulation of OPG in the supernatants of these neutrophils $(2.0 \pm 0.4 \mathrm{pg} / \mathrm{ml}, n=7)$. Moreover, the same neutrophil supernatants contained no RANK-L as evaluated by EIA (data not shown). In contrast, PBMLs cultured under similar conditions secreted RANK-L and OPG in the supernatants (data not shown), confirming that neutrophils and PBMLs expressed RANK-L and OPG differently.

\section{SF from patients with RA activates the expression of RANK-L, OPG, and RANK in normal blood neutrophils}

Having established that inflammatory cytokines can stimulate healthy blood neutrophils to express OPG and RANK (Figure 3) and that SF neutrophils from patients with RA spontaneously expressed RANK-L, OPG, and RANK proteins (Figure 2), we next investigated the effect of SF on the expression of these genes by incubating healthy human blood neutrophils in the presence of SF from patients with RA (Figure 4). After 2 days of incubation of healthy blood neutrophils in medium containing $80 \%$ SF from patients with RA and $20 \%$ $\mathrm{CM}$, membrane RANK-L significantly increased and was detected on $13.4 \% \pm 4.7 \%$ of cells $(n=5)$ (versus $2.5 \% \pm$ $0.8 \%$ neutrophils in $\mathrm{CM}$ alone, a percentage similar to that of freshly isolated neutrophils). Similar experiments with incubation medium containing SF from patients with OA revealed that $3.9 \% \pm 0.5 \%$ of neutrophils expressed membrane RANK$\mathrm{L}(n=14)$ (Figure $4 \mathrm{a})$. The difference in the expression of membrane RANK-L in healthy blood neutrophils incubated in SF from patients with RA versus patients with OA was significant $(p=0.019)$. Moreover, SF from patients with RA, but not patients with $\mathrm{OA}$, activated healthy blood neutrophils to express OPG and RANK mRNAs as evaluated by RT-PCR (Figure 4b). Finally, SFs from patients with RA, but not from patients with $\mathrm{OA}$, strongly activated healthy blood neutrophils to express RANK at the cell surface. Membrane RANK, which is not expressed by freshly isolated human blood neutrophils (data not shown), was detected on $15.3 \% \pm 5.6 \%$ of cells after 3 days of incubation in the presence of SF of patients with RA (Figure 4c).

The release of active nuclear factor-kappa-B (NF- $\kappa B$ ) secondary to the stimulation of RANK by RANK-L is associated with the phosphorylation of the inhibitory $\mathrm{I}-\kappa \mathrm{B}-\alpha$ protein. Subsequently, I- $\mathrm{KB}-\alpha$ decreased through its conjugation with ubiquitin and its degradation by proteasome. To determine whether 


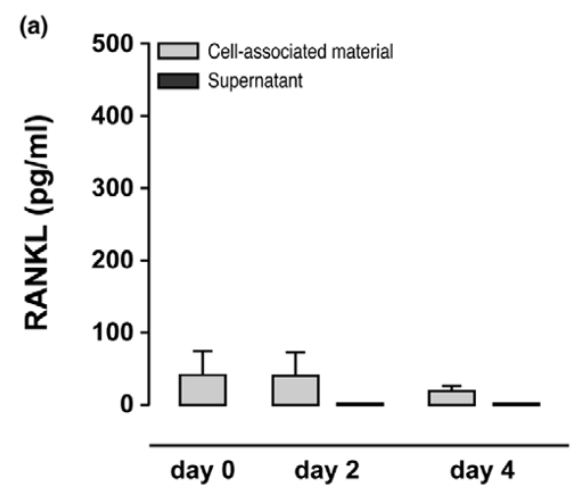

(b)
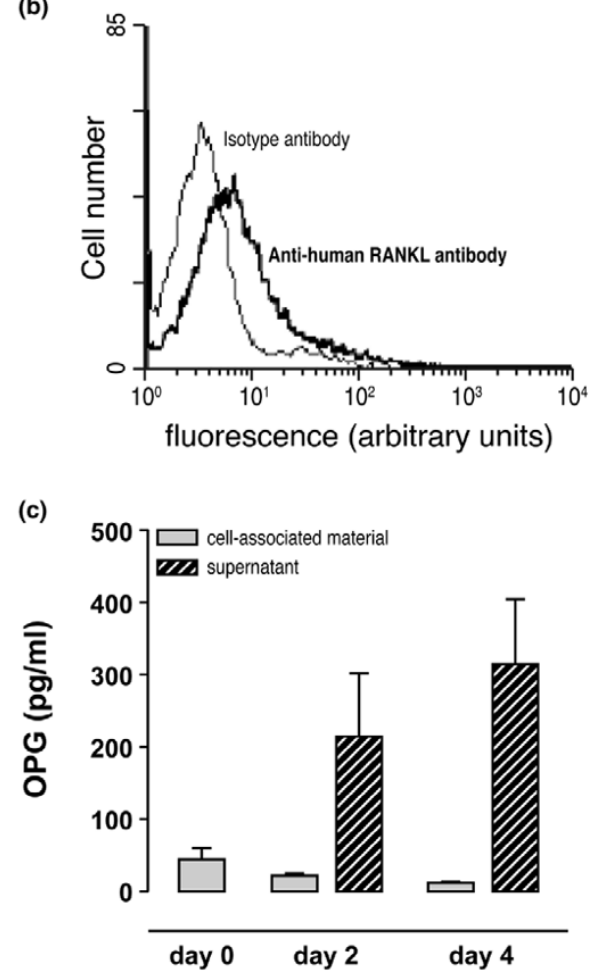

(d)

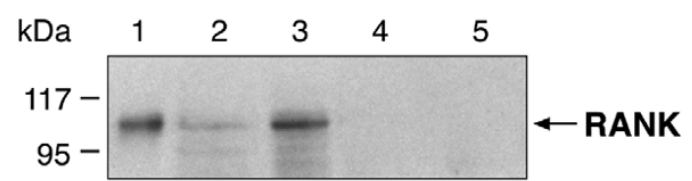

Expression of RANK-L, OPG, and RANK proteins by synovial fluid (SF) neutrophils from patients with rheumatoid arthritis (RA). (a) Cell-associated materials and supernatants of SF neutrophils $\left(10^{7} / \mathrm{ml}\right)$ from three patients with RA were analyzed by enzyme immunometric assays (EIAs) for RANK$L$ at day 0 and after 2 and 4 days of incubation in control medium. (b) Surface expression of RANK-L by freshly isolated SF neutrophils from patients with RA. Flow cytometry was performed after incubation of neutrophils with a goat anti-human RANK-L antibody followed by a fluorescein isothiocyanate-conjugated anti-goat $F\left(a b^{\prime}\right)_{2}$ antibody. Control isotype antibody was a normal goat immunoglobulin $G$ (lgG). Results shown are representative of SF neutrophils from three patients with RA. (c) Samples similar to those in (a) were analyzed by EIAs for OPG. (d) Cell-associated materials of SF neutrophils from four patients with RA were solubilized in SDS sample buffer and subjected to SDS-PAGE under reducing conditions (lanes 2 to 5). Protein loading values in lanes $2,3,4$, and 5 were $123,163,135$, and $125 \mu \mathrm{g}$, respectively. COS-7 cells transfected with a human RANK cDNA were used as a positive control (lane 1). Western blotting was performed with a goat anti-human RANK antibody, a horseradish peroxidase-conjugated anti-goat lgG antibody, and the enhanced chemiluminescence detection system. The position of the molecular weight markers in kilodaltons is indicated on the left. OPG, osteoprotegerin; RANK, receptor activator of nuclear factor-kappa-B; RANK-L, ligand of receptor activator of nuclear factor-kappa-B. 


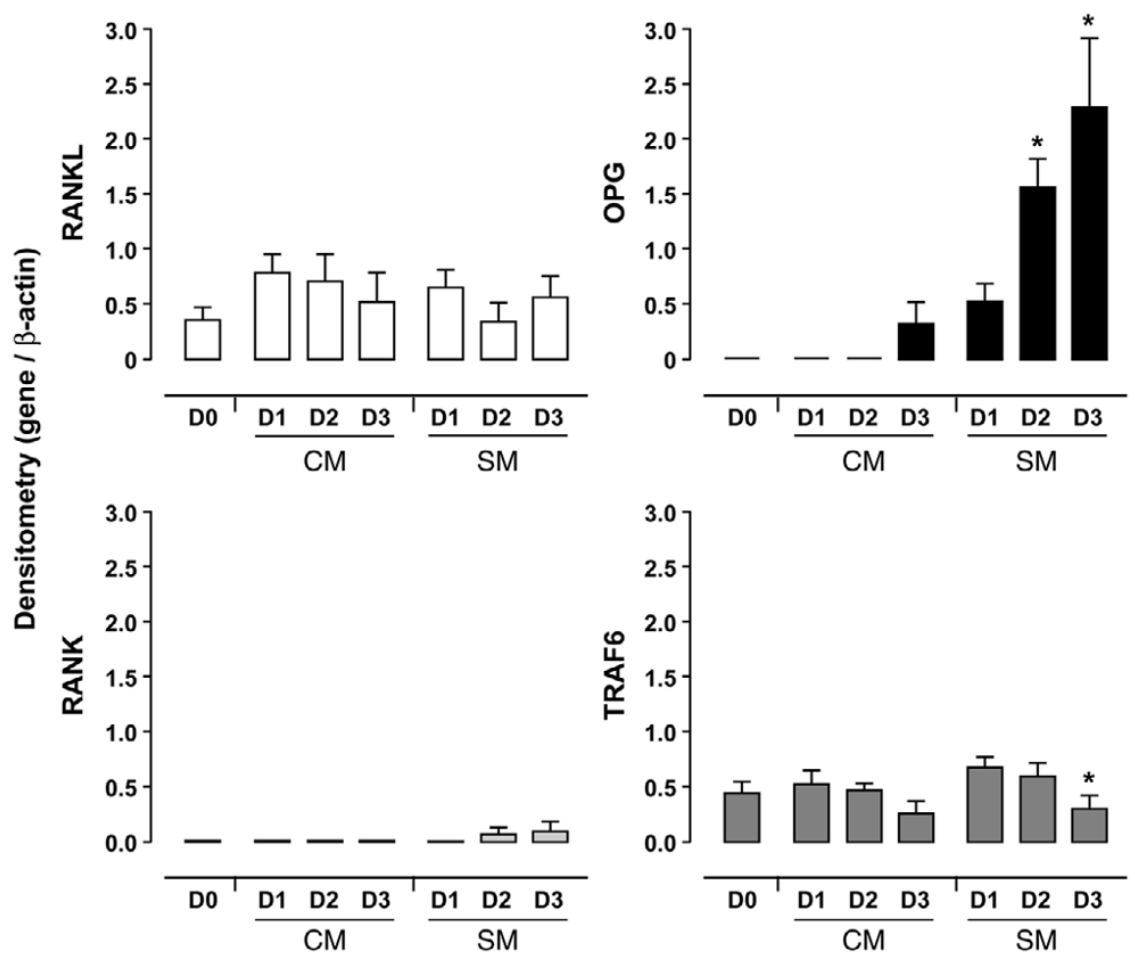

Effect of cytokines on the expression of RANK-L, OPG, RANK, and TRAF6 mRNAs by normal human blood neutrophils in vitro. Purified neutrophils were incubated in control medium (CM) or in survival medium (SM) for 1 to 3 days (D1, D2, D3). After RNA extraction, semi-quantitative reverse transcriptase-polymerase chain reaction analysis was performed. Histograms represent mean \pm standard error of the mean of ratios of densitometric values for RANK-L/ $\beta$-actin, OPG/ $\beta$-actin, RANK/ $\beta$-actin, and TRAF6/ $\beta$-actin ( $n=5$ normal donors). Student's paired $t$ test: ${ }^{*} p<0.05$ (D3 versus D1, D2 versus D1). OPG, osteoprotegerin; RANK, receptor activator of nuclear factor-kappa-B; RANK-L, ligand of receptor activator of nuclear factorkappa-B; TRAF6, tumor necrosis factor receptor-associated factor 6.

RANK expressed on the surface of neutrophils was functional, healthy blood neutrophils preincubated 3 days in SF from patients with RA were stimulated by RANK-L (or TNF- $\alpha$ as a positive control) and total amounts of $\mathrm{I}-\kappa \mathrm{B}-\alpha$ protein were evaluated by Western blotting. A time-dependent decrease of I$\kappa B-\alpha$ protein was demonstrated in the presence of RANK-L or TNF- $\alpha$ (Figure 5), confirming that the stimulation of cell surface RANK in neutrophils pretreated with SF from patients with RA was followed by intracellular signaling through, at least in part, the NF-кB pathway.

\section{Discussion}

The present report is the first to demonstrate that neutrophils have the capacity to express proteins of the RANK pathway. We observed the expression of the membrane-associated form of RANK-L in healthy blood neutrophils. In contrast, SF neutrophils from patients with RA not only express the membrane-associated form of RANK-L but also express RANK and secrete OPG. Remarkably, healthy human blood neutrophils can be induced to express RANK and OPG in response to different stimuli such as IL-4+TNF- $\alpha$ and SF from patients with RA. The RANK protein expressed on the surface of neutrophils stimulated by SF from patients with RA is functional since it can be activated in the presence of RANK-L. Interestingly,
TRAF6 is expressed by both inflammatory and healthy neutrophils and its expression is not modulated by any stimulus. These findings may have important pathophysiological implications considering that neutrophils are present in large numbers at inflammatory sites and are involved in cell-cell interactions in inflamed tissues.

The fact that SF and blood neutrophils express RANK-L as membrane materials and that neutrophils incubated in vitro for up to 4 days generated no soluble RANK-L (Figure 2a) allow us to consider neutrophils as a new cell type that generates RANK-L without any release in the extracellular milieu. From that point of view, neutrophils are different from other cell types such as osteoblasts, fibroblasts, or T lymphocytes, which produce RANK-L and release soluble RANK-L after stimulation [17,24,30,31]. In the context of a chronic inflammatory reaction, RANK-L/RANK interactions between T lymphocytes and dendritic cells and between $T$ lymphocytes and osteoclasts explain the role of $\mathrm{T}$ cells in disease progression $[17,24,32]$. The enhancing effect of SF from patients with RA on the expression of neutrophil membrane RANK-L (Figure 4a) should not be neglected in terms of cell-cell interactions. Neutrophils have been described at sites of rheumatoid pannus invasion into cartilage and subchondral bone [5]. Thus, infil- 
(a)

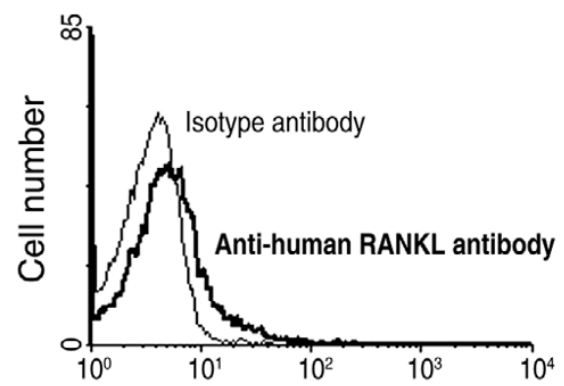

\section{Blood neutrophils in OA-SF}

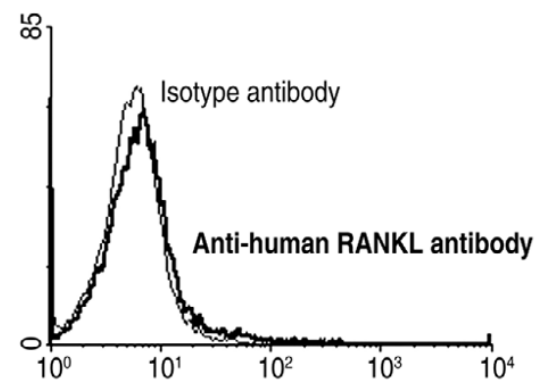

fluorescence (arbitrary units)

(b)

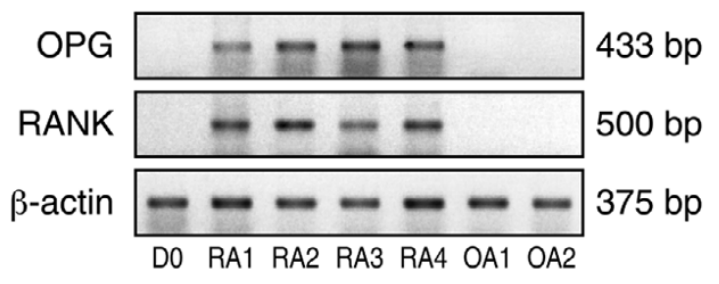

(c)

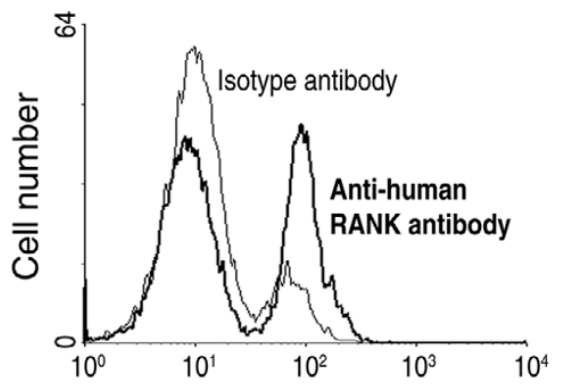

\section{Blood neutrophils in OA-SF}

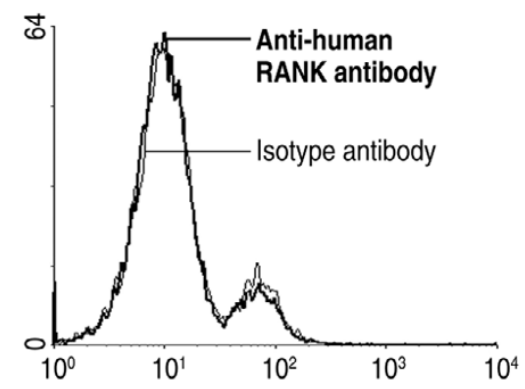

fluorescence (arbitrary units)

Induction of the expression of RANK-L, OPG, and RANK by normal human blood neutrophils incubated in the presence of synovial fluid (SF) from patients with rheumatoid arthritis (RA) or patients with osteoarthritis (OA). (a) Surface expression of RANK-L by normal blood neutrophils incubated in SF from patients with RA (RA-SF) or OA (OA-SF) for 2 days. Flow cytometry was performed after incubation of neutrophils with a goat anti-human RANK-L antibody followed by a fluorescein isothiocyanate (FITC)-conjugated anti-goat $F(a b ')_{2}$ antibody. Control isotype antibody was a normal goat immunoglobulin G (lgG). Results shown are representative of three RA-SF and nine OA-SF. (b) Expression of mRNA for OPG and RANK by normal blood neutrophils incubated for 2 days in SF from four patients with RA and two patients with OA. Total RNA was isolated from freshly isolated normal blood neutrophils (D0) and from neutrophils of the same healthy donors after 2 days of incubation in SF (RA-1 to -4, OA-1, -2). RNA was then analyzed by reverse transcriptase-polymerase chain reaction. Results shown are representative of two different healthy donors. (c) Surface expression of RANK by normal blood neutrophils incubated in SF from patients with RA (RA-SF) or OA (OA-SF) for 3 days. Flow cytometry was performed after cellular fixation, permeabilization, and staining with a mouse monoclonal anti-human RANK antibody followed by a FITC-conjugated anti-mouse $\mathrm{F}\left(a b^{\prime}\right)_{2}$ antibody. Control isotype antibody was a non-specific mouse lgG. Results shown are representative of neutrophils from two different healthy subjects incubated in three different RA-SF and two OA-SF. OPG, osteoprotegerin; RANK, receptor activator of nuclear factor-kappa-B; RANK-L, ligand of receptor activator of nuclear factor-kappa-B.

trating neutrophils that, therefore, are numerous and implicated in the local inflammatory process of active immune diseases could also directly impact on the local immune and bone remodeling responses through their membrane RANK-L.
The rheumatoid pannus-bone junction at sites of subchondral bone destruction showed local RANK-L expression that was more prevalent in active RA [33]. The cellular sources of RANK-L in these rheumatoid bone destruction sites were not 


\section{Figure 5}

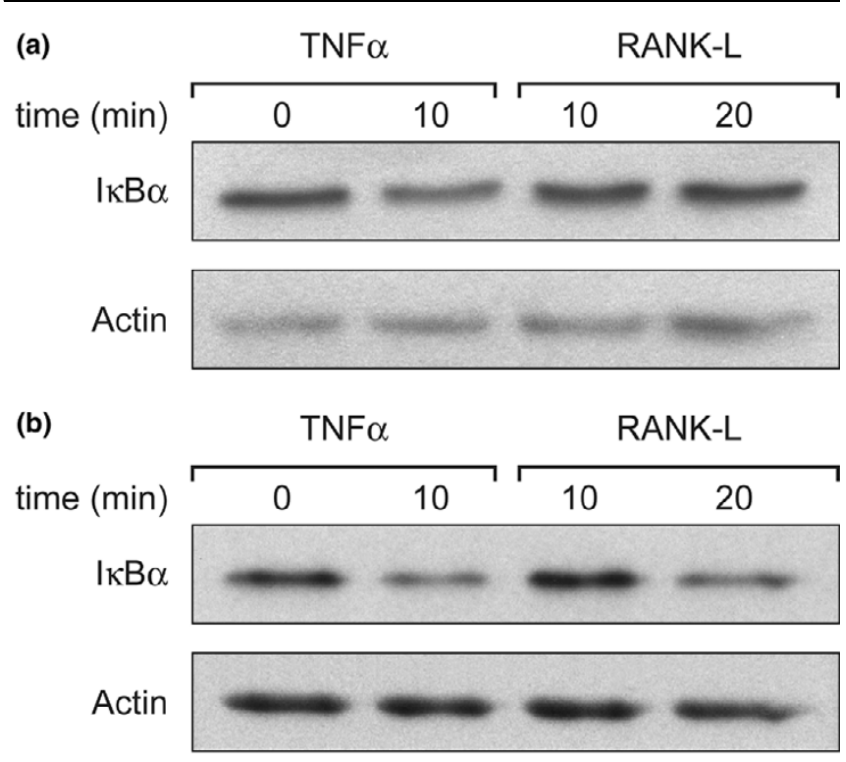

Degradation of inhibitor of kappaB-alpha (I-KB- $\alpha)$ in RANK-L-activated neutrophils. I- $\mathrm{KB}-\alpha$ was detected in whole-cell lysates by Western blotting as described in Materials and methods. Freshly isolated neutrophils (a), or blood neutrophils pretreated for a 3-day incubation period in rheumatoid arthritis-synovial fluid (RA-SF) $(80 \%)+$ control medium (20\%) (b), were stimulated with $50 \mathrm{ng} / \mathrm{ml}$ tumor necrosis factor-alpha (TNF- $\alpha$ ) for 10 minutes or with $100 \mathrm{ng} / \mathrm{ml}$ RANK-L for 10 and 20 minutes. Western blotting was performed with a rabbit anti-human I$\kappa \mathrm{B}-\alpha$ antibody, a horseradish peroxidase-conjugated anti-rabbit immunoglobulin $\mathrm{G}$ antibody, and the enhanced chemiluminescence detection system. Results shown are representative of neutrophils from three different healthy subjects incubated in three different RA-SF. RANK-L, ligand of receptor activator of nuclear factor-kappa-B.

all identified with no mention of neutrophils [33]. The present data on the increased RANK-L expression by RA neutrophils, together with the presence of neutrophils at the pannus-bone interface [34], suggest that through cell-cell interactions such inflammatory neutrophils could activate RANK-expressing osteoclasts and bone resorption.

The capacity of neutrophils freshly isolated from inflammatory SFs to express large quantities of OPG (Figures 1a and 2c) in comparison to the inferior amount of OPG expressed by healthy blood neutrophils after incubation with certain stimuli (Figure 3) suggests that the induction of OPG expression by neutrophils is regulated by multiple factors. In vitro, the maximal concentration of OPG released by neutrophils in the presence of IL-4, TNF- $\alpha$, and GM-CSF was approximately $2 \mathrm{pg} / \mathrm{ml}$. In contrast, OPG concentrations spontaneously released in supernatants of SF neutrophils were 200 to $300 \mathrm{pg} / \mathrm{ml}$. It follows that, if the cytokine combination of IL-4, TNF- $\alpha$, and GMCSF cannot induce neutrophils to express the high concentrations of OPG observed with neutrophils from patients with RA, other factors are involved in inducing OPG. The effect of IL-4 on neutrophil expression of OPG, however, could be associated with the anti-apoptotic function of IL-4 through OPG inhi- bition of TRAIL (tumor necrosis factor-related apoptosisinducing ligand) produced by human neutrophils [35,36]. A synergism between IL- 4 and TNF- $\alpha$ has been demonstrated for the increased production of IL-1 receptor antagonist in human neutrophils [37]. The exact mechanism (or mechanisms) underlying the synergism that stimulates the neutrophil expression of OPG remains to be elucidated and could be independent of or complementary to the NF- $\mathrm{BB}$ pathway that is simultaneously activated by IL-4 and by TNF- $\alpha[38,39]$. Moreover, IL-4 not only activates human blood neutrophils but also is a maturation factor for precursors to become neutrophils [40] and could drive a subpopulation of neutrophils and some of their precursors present in blood to express OPG. The high concentrations of OPG measured in SF from patients with RA (see Results section) could be related to the capacity of neutrophils, which are present in large numbers, to release OPG (Figure 2c). On the other hand, given that RANK$L$ was not released by inflammatory neutrophils (Figure $2 a$ ), the low amounts of RANK-L measured in the same SF (see Results section) could originate only from lining fibroblast-like synoviocytes [41].

The findings that inflammatory neutrophils spontaneously express RANK (Figures 1 and $2 \mathrm{~d}$ ) and that healthy blood neutrophils express RANK only after stimulation raise the possibility that neutrophils are involved in bone remodeling. However, compared with the cytokine combination present in SM, the SFs from patients with RA are more efficient at activating neutrophils to express RANK, indicating that factors other than GM-CSF+IL-4+TNF- $\alpha$ are implicated in inducing RANK expression. The production of RANK protein by inflammatory neutrophils could be related to a pathophysiological role. The presence of a functional RANK protein at the cell surface of neutrophils pretreated by SFs from patients with RA, as demonstrated by RANK-L activation of the NF- $\kappa B$ pathway (Figure 5 ), indicates that such neutrophils contribute to the local tissue response.

Our findings that inflammatory neutrophils from rheumatoid SF expressed RANK at the mRNA and protein levels further confirm the plasticity of neutrophils during inflammation. Similar results were obtained with neutrophils from SF of patients with psoriatic arthritis (PE Poubelle, unpublished observations). Neutrophils can acquire the functional phenotype of active dendritic cells $[10,11]$. Mature dendritic cells express RANK $[15,16]$. Thus, the demonstration that inflammatory neutrophils express RANK could be related, in part, to their capacity of acquiring the functional phenotype of active dendritic cells, as reported in RA or Wegener granulomatosis $[9,10,42,43]$. The exact functions associated with neutrophil expression of RANK, however, remain to be elucidated. It is of note that neutrophil-neutrophil and neutrophil-T lymphocyte interactions have been described in pathophysiological situations [44]. Moreover, activated neutrophils have several characteristics of bone-resorbing cells. These characteristics include the capac- 
ity to form a ruffled border and the combined expression of $\alpha_{v} \beta_{3}$ integrin and of certain enzymes (carbonic anhydrase II, vacuolar ATPase, cathepsin). This lends support to the hypothesis that neutrophils could be involved in bone remodeling.

The constant expression of TRAF 6 by healthy and inflammatory neutrophils (Figures 1 and 3 ) suggests that this cytoplasmic adapter protein, which is required for immunity and bone homeostasis, is not a limiting factor in RANK-mediated neutrophil effector functions. The present report is the first to describe TRAF6 expression by neutrophils. These cells have been found to delay their programmed cell death induced by TNF- $\alpha$ through NF- $\kappa B$ and TRAF1 induction [45]. Investigation of neutrophil functions linked to TRAF6 will further our understanding of the role of this adapter protein in neutrophil biology.

\section{Conclusion}

Direct evidence is provided for the differential expression of proteins of the RANK/RANK-L pathway in neutrophils in a noninflammatory versus inflammatory context. Moreover, signaling occurs via RANK, the expression of which is induced in stimulated neutrophils. The results of the present study, therefore, suggest that neutrophils could play a dual role as immune and bone-like cells during the inflammatory process. This could occur via a direct interaction with dendritic cells or osteoclasts mediated by their membrane RANK-L. In certain inflammatory conditions, these neutrophils could be directly involved in acquired immunity or in bone remodeling through their expression of RANK, depending on the factors present simultaneously at the inflammatory site. As has been proposed for monocyte/macrophage precursor cells that can be driven to differentiate into dendritic cells or osteoclasts, the acquired changes of neutrophils reported above could represent an intermediary phenotype observed during the transdifferentiation of these cells [46].

\section{Competing interests}

The authors declare that they have no competing interests.

\section{Authors' contributions}

PEP conceived of the study, designed experiments, evaluated data, and wrote the manuscript. AC participated in the design of the study, performed experiments, and evaluated data. MJF participated in the design of the study and helped to draft the manuscript. KD performed experiments that involved molecular biology and evaluated data. A-AM carried out the immunoassays and evaluated data. All authors read and approved the final manuscript.

\section{Acknowledgements}

We thank Marie-Lisane Tremblay for her excellent technical assistance. This work was supported by grants from the Canadian Institutes for Health Research.

\section{References}

1. Lipsky PE, Davis LS, Cush JJ, Oppenheimer-Marks N: The role of cytokines in the pathogenesis of rheumatoid arthritis. Springer Semin Immunopathol 1989, 11:123-162.

2. Duan H, Koga T, Kohda F, Hara H, Urabe K, Furue M: Interleukin8-positive neutrophils in psoriasis. J Dermatol Sci 2001, 26:119-124.

3. Mitsuyama K, Toyonaga A, Sasaki E, Watanabe K, Tateishi H, Nishiyama T, Saiki T, lkeda H, Tsuruta O, Tanikawa K: IL-8 as an important chemoattractant for neutrophils in ulcerative colitis and Crohn's disease. Clin Exp Immunol 1994, 96:432-436.

4. Chatham WW, Swaim R, Frohsin H Jr, Heck LW, Miller EJ, Blackburn WD Jr: Degradation of human articular cartilage by neutrophils in synovial fluid. Arthritis Rheum 1993, 36:51-58.

5. Mohr W, Westerhellweg H, Wessinghage D: Polymorphonuclear granulocytes in rheumatic tissue destruction. III. an electron microscopic study of PMNs at the pannus-cartilage junction in rheumatoid arthritis. Ann Rheum Dis 1981, 40:396-399.

6. Wipke BT, Allen PM: Essential role of neutrophils in the initiation and progression of a murine model of rheumatoid arthritis. J Immunol 2001, 167:1601-1608.

7. Ashtekar AR, Saha B: Poly's plea: membership to the club of APCs. Trends Immunol 2003, 24:485-490.

8. Gosselin EJ, Wardwell K, Rigby WF, Guyre PM: Induction of MHC class II on human polymorphonuclear neutrophils by granulocyte/macrophage colony-stimulating factor, IFN-gamma, and IL-3. J Immunol 1993, 151:1482-1490.

9. Radsak M, Iking-Konert C, Stegmaier S, Andrassy K, Hansch GM: Polymorphonuclear neutrophils as accessory cells for T-cell activation: major histocompatibility complex class II restricted antigen-dependent induction of T-cell proliferation. Immuno/ogy 2000, 101:521-530.

10. Oehler L, Majdic O, PickI WF, Stockl J, Riedl E, Drach J, Rappersberger K, Geissler K, Knapp W: Neutrophil granulocyte-committed cells can be driven to acquire dendritic cell characteristics. $J$ Exp Med 1998, 187:1019-1028.

11. Yamashiro $S$, Wang JM, Yang $D$, Gong WH, Kamohara $H$, Yoshimura T: Expression of CCR6 and CD83 by cytokine-activated human neutrophils. Blood 2000, 96:3958-3963.

12. Iking-Konert C, Vogt S, Radsak M, Wagner C, Hansch GM, Andrassy K: Polymorphonuclear neutrophils in Wegener's granulomatosis acquire characteristics of antigen presenting cells. Kidney Int 2001, 60:2247-2262.

13. Cross A, Bucknall RC, Cassatella MA, Edwards SW, Moots RJ: Synovial fluid neutrophils transcribe and express class II major histocompatibility complex molecules in rheumatoid arthritis. Arthritis Rheum 2003, 48:2796-2806.

14. Colotta F, Re F, Polentarutti N, Sozzani S, Mantovani A: Modulation of granulocyte survival and programmed cell death by cytokines and bacterial products. Blood 1992, 80:2012-2020.

15. Anderson DM, Maraskovsky E, Billingsley WL, Dougall WC, Tometsko ME, Roux ER, Teepe MC, DuBose RF, Cosman D, Galibert L: A homologue of the TNF receptor and its ligand enhance Tcell growth and dendritic-cell function. Nature 1997, 390:175-179.

16. Ye H, Arron JR, Lamothe B, Cirilli M, Kobayashi T, Shevde NK, Segal D, Dzivenu OK, Vologodskaia M, Yim M, et al.: Distinct molecular mechanism for initiating TRAF6 signalling. Nature 2002, 418:443-447.

17. Wong BR, Josien R, Lee SY, Sauter B, Li HL, Steinman RM, Choi $Y$ : TRANCE (tumor necrosis factor [TNF]-related activationinduced cytokine), a new TNF family member predominantly expressed in T cells, is a dendritic cell-specific survival factor. $J$ Exp Med 1997, 186:2075-2080.

18. Cremer I, Dieu-Nosjean MC, Marechal S, Dezutter-Dambuyant C, Goddard S, Adams D, Winter N, Menetrier-Caux C, Sautes-Fridman $\mathrm{C}$, Fridman $\mathrm{WH}$, et al:: Long-lived immature dendritic cells mediated by TRANCE-RANK interaction. Blood 2002, 100:3646-3655.

19. Lacey DL, Timms E, Tan HL, Kelley MJ, Dunstan CR, Burgess T, Elliott R, Colombero A, Elliott G, Scully S, et al.: Osteoprotegerin ligand is a cytokine that regulates osteoclast differentiation and activation. Cell 1998, 93:165-176.

20. Yasuda $H$, Shima N, Nakagawa N, Yamaguchi $K$, Kinosaki $M$, Mochizuki S, Tomoyasu A, Yano K, Goto M, Murakami A, et al.: Osteoclast differentiation factor is a ligand for osteoprote- 
gerin/osteoclastogenesis-inhibitory factor and is identical to TRANCE/RANKL. Proc Natl Acad Sci USA 1998, 95:3597-3602

21. Burgess TL, Qian Y, Kaufman S, Ring BD, Van G, Capparelli C, Kelley M, Hsu H, Boyle WJ, Dunstan CR, et al:: The ligand for osteoprotegerin (OPGL) directly activates mature osteoclasts. J Cell Biol 1999, 145:527-538.

22. Lum L, Wong BR, Josien R, Becherer JD, Erdjument-Bromage $\mathrm{H}$, Schlondorff J, Tempst P, Choi Y, Blobel CP: Evidence for a role of a tumor necrosis factor-alpha (TNF-alpha)-converting enzyme-like protease in shedding of TRANCE, a TNF family member involved in osteoclastogenesis and dendritic cell survival. J Biol Chem 1999, 274:13613-13618.

23. Wang R, Zhang L, Zhang X, Moreno J, Celluzzi C, Tondravi M, Shi $Y$ : Regulation of activation-induced receptor activator of NFkappaB ligand (RANKL) expression in T cells. Eur J Immunol 2002, 32:1090-1098.

24. Kong YY, Feige U, Sarosi I, Bolon B, Tafuri A, Morony S, Capparelli $\mathrm{C}, \mathrm{Li}$ J, Elliott R, McCabe S, et al.: Activated T cells regulate bone loss and joint destruction in adjuvant arthritis through osteoprotegerin ligand. Nature 1999, 402:304-309.

25. Boyum A: Isolation of mononuclear cells and granulocytes from human blood. Isolation of monuclear cells by one centrifugation, and of granulocytes by combining centrifugation and sedimentation at 1 g. Scand J Clin Lab Invest Supp/ 1968, 97:77-89.

26. Brach MA, deVos S, Gruss HJ, Herrmann F: Prolongation of survival of human polymorphonuclear neutrophils by granulocyte-macrophage colony-stimulating factor is caused by inhibition of programmed cell death. Blood 1992, 80:2920-2924.

27. Zamorano J, Wang HY, Wang LM, Pierce JH, Keegan AD: IL-4 protects cells from apoptosis via the insulin receptor substrate pathway and a second independent signaling pathway. $J$ Immunol 1996, 157:4926-4934.

28. Ren Y, Stuart L, Lindberg FP, Rosenkranz AR, Chen Y, Mayadas TN, Savill J: Nonphlogistic clearance of late apoptotic neutrophils by macrophages: efficient phagocytosis independent of beta 2 integrins. J Immunol 2001, 166:4743-4750.

29. Raza K, Falciani F, Curnow SJ, Ross EJ, Lee CY, Akbar AN, Lord JM, Gordon C, Buckley CD, Salmon M: Early rheumatoid arthritis is characterized by a distinct and transient synovial fluid cytokine profile of $\mathrm{T}$ cell and stromal cell origin. Arthritis Res Ther 2005, 7:R784-795.

30. Theill LE, Boyle WJ, Penninger JM: RANK-L and RANK: T cells, bone loss, and mammalian evolution. Annu Rev Immunol 2002 , 20:795-823.

31. Kanamaru F, Iwai H, Ikeda T, Nakajima A, Ishikawa I, Azuma M: Expression of membrane-bound and soluble receptor activator of NF-kappaB ligand (RANKL) in human T cells. Immunol Lett 2004, 94:239-246.

32. Josien R, Li HL, Ingulli E, Sarma S, Wong BR, Vologodskaia M, Steinman RM, Choi Y: TRANCE, a tumor necrosis factor family member, enhances the longevity and adjuvant properties of dendritic cells in vivo. J Exp Med 2000, 191:495-502.

33. Pettit AR, Walsh NC, Manning C, Goldring SR, Gravallese EM: RANKL protein is expressed at the pannus-bone interface at sites of articular bone erosion in rheumatoid arthritis. Rheumatology (Oxford) 2006, 45:1068-1076.

34. Mohr W, Menninger $\mathrm{H}$ : Polymorphonuclear granulocytes at the pannus-cartilage junction in rheumatoid arthritis. Arthritis Rheum 1980, 23:1413-1414.

35. Cassatella MA: On the production of TNF-related apoptosisinducing ligand (TRAIL/Apo-2L) by human neutrophils. J Leukoc Biol 2006, 79:1140-1149.

36. Emery JG, McDonnell P, Burke MB, Deen KC, Lyn S, Silverman C, Dul E, Appelbaum ER, Eichman C, DiPrinzio R, et al.: Osteoprotegerin is a receptor for the cytotoxic ligand TRAIL. J Biol Chem 1998, 273:14363-14367.

37. Marie C, Pitton C, Fitting C, Cavaillon JM: IL-10 and IL-4 synergize with TNF-alpha to induce IL-1ra production by human neutrophils. Cytokine 1996, 8:147-151.

38. Zamorano J, Mora AL, Boothby M, Keegan AD: NF-kappa B activation plays an important role in the IL-4-induced protection from apoptosis. Int Immunol 2001, 13:1479-1487.
39. McDonald PP, Bald A, Cassatella MA: Activation of the NF-kappaB pathway by inflammatory stimuli in human neutrophils. Blood 1997, 89:3421-3433.

40. Bober LA, Waters TA, Pugliese-Sivo CC, Sullivan LM, Narula SK, Grace MJ: IL-4 induces neutrophilic maturation of HL-60 cells and activation of human peripheral blood neutrophils. Clin Exp Immunol 1995, 99:129-136.

41. Shigeyama Y, Pap T, Kunzler P, Simmen BR, Gay RE, Gay S: Expression of osteoclast differentiation factor in rheumatoid arthritis. Arthritis Rheum 2000, 43:2523-2530.

42. Fanger NA, Liu C, Guyre PM, Wardwell K, O'Neil J, Guo TL, Christian TP, Mudzinski SP, Gosselin EJ: Activation of human T cells by major histocompatibility complex class II expressing neutrophils: proliferation in the presence of superantigen, but not tetanus toxoid. Blood 1997, 89:4128-4135.

43. Iking-Konert C, Ostendorf B, Sander O, Jost M, Wagner C, Joosten L, Schneider M, Haensch MG: Trans-differentiation of polymorphonuclear neutrophils to dendritic-like cells in the synovial fluid in rheumatoid arthritis: evidence for activation by T-cells. Ann Rheum Dis 2005, 64:1436-1442.

44. Zhang JH, Ferrante A, Arrigo AP, Dayer JM: Neutrophil stimulation and priming by direct contact with activated human $\mathrm{T}$ lymphocytes. J Immunol 1992, 148:177-181.

45. Nolan B, Kim R, Duffy A, Sheth K, De M, Miller C, Chari R, Bankey $\mathrm{P}$ : Inhibited neutrophil apoptosis: proteasome dependent NFkappaB translocation is required for TRAF-1 synthesis. Shock 2000, 14:290-294.

46. Rivollier A, Mazzorana M, Tebib J, Piperno M, Aitsiselmi T, Rabourdin-Combe C, Jurdic P, Servet-Delprat C: Immature dendritic cell transdifferentiation into osteoclasts: a novel pathway sustained by the rheumatoid arthritis microenvironment. Blood 2004, 104:4029-4037. 